KYUNGPOOK Math. J. 52(2012), 327-346

http://dx.doi.org/10.5666/KMJ.2012.52.3.327

\title{
A New Time Stepping Method for Solving One Dimensional Burgers' Equations
}

\author{
Xiang Fan Piao, SangDong Kim and Philsu Kim* \\ Department of Mathematics, Kyungpook National University, Daegu, 702-701, Ko- \\ rea \\ e-mail : piaoxf76@gmail.com, skim@knu.ac.kr, and kimps@knu.ac.kr \\ Do-Hyung Kim \\ Department of Physics, Kyungpook National University, Daegu, 702-701, Korea \\ e-mail : kimdh@knu.ac.kr
}

ABSTRACT. In this paper, we present a simple explicit type numerical method for discretizations in time for solving one dimensional Burgers' equations. The proposed method does not need an iteration process that may be required in most implicit methods and have good convergence and efficiency in computational sense compared to other known numerical methods. For evidences, several numerical demonstrations are also provided.

\section{Introduction}

Over the past decades, many numerical techniques have been developed for solving Burgers' equations because of its importance in many areas such as gas dynamics, acoustic and turbulence phenomena, etc (see $[5,7,25]$ ). For example, one may refer to the approaches based on the finite difference method $([2,12,16,18,20,23,27])$, Galerkin method $([11,17,33])$, finite element method $([1,8,10,19,24,28])$, spectral method $([4,6,26])$ and cubic spline and sinc-function methods $([1,24,31,32]$ etc. Most of the mentioned numerical approaches are using various computational techniques in spatial discretizations to get more accurate approximations. The main issues in developing numerical algorithms are to reduce the computational costs and to enhance the accuracy by all possible means. These issues usually occur from three representative aspects of Burgers' equations such as small viscosity, stiffness and nonlinearity.

It is well known that the small viscosity leads to a steep gradient for the so-

* Corresponding Author.

Received April 6, 2011; accepted September 23, 2011.

2010 Mathematics Subject Classification: 65N35, 65L04, 65L07.

Key words and phrases: Error corrected Euler method; Pseudo-Spectral method.

This work was supported by basic science research program through the National Research

Foundation of Korea(NRF) funded by the ministry of education, science and technology (grant number 2011-0029013) 
lution of Burgers' equation in the spatial domain and requires either a high order approximation or quite small mesh length in space for a highly accurate approximation. The spatial discretization may lead to a first order nonlinear system of ordinary differential equations (ODE) in time with a large scale, which are usually very stiff (see Section 2.1). It is known that all standard explicit type methods of convergence order 2 and larger are not sufficient to overcome the stiffness difficulty [14]. On the other hand, all efficient and popular implicit type methods may require extra computations comparing to explicit methods because of the nonlinearity of the Burgers' equation.

In this paper, we will use the popular and very accurate pseudo-spectral method $[9,30]$ for the spatial domain so that one may have a nonlinear stiff system in time. Because of the accuracy of spacial discretizations, we may require an accurate time approximations for solving a nonlinear stiff system in time. Hence the primary goal of the present paper is to construct a non-standard type of an explicit method providing a nice spatial discretization, which does not ask to solve any extra nonlinear equations required by an implicit method. The error corrected Euler method (ECEM) with convergence order 4 for the scalar stiff initial value problem developed by authors [22] is extended to solve a system of ODEs in this paper. To confirm the effectiveness and convergence, the present method is compared with several known methods. In particular, the numerical results through several test problems show that the present method radically reduce about 50 percent of the computational cost in the sense of function evaluations compared with the fourth-order implicit type Runge-Kutta method based on BDF-type Chebyshev approximation [29].

The rest of paper is organized as follows. In section 2, the pseudo-spectral method is applied to Burgers' equations for spacial approximations which leads to a system of ODEs in time. The stiffness ratios for the model Burgers' equation is also discussed. The error corrected Euler's algorithm (ECEM) for the general system of ODEs is presented in the section 3. Numerical tests for periodic and nonperiodic Burgers' examples are demonstrated in section 4. Finally, the conclusion of this work with some comments is provided in the last section.

\section{Space discretizations}

The target problem we consider in this paper is the one-dimensional Burgers' problem of the form

$$
\left\{\begin{aligned}
\frac{\partial u}{\partial t}+u \frac{\partial u}{\partial x} & =\epsilon \frac{\partial^{2} u}{\partial x^{2}}, & & a<x<b, \quad 0<t \leq t_{\text {end }}, \\
u(0, x) & =Q(x), & & a \leq x \leq b, \\
u(t, a)=q_{1}(t), u(t, b) & =q_{2}(t), & & 0<t \leq t_{\text {end }},
\end{aligned}\right.
$$

where $\epsilon$ is the given viscosity and $Q(x)$ and $q_{i}(t), i=1,2$ are given initial and boundary conditions, respectively. Note that the solution $u(t, x)$ of $(2.1)$ develops shock waves when $\epsilon$ approaches to 0 . For spatial discretizations of (2.1), the pseudospectral approximation will be used because it is known as one of the accurate and 
popular methods among other numerical methods (see $[9,15,21]$ for example). For a positive integer $N$, let $\left\{x_{j}\right\}_{j=0}^{N+1}$ be the Chebyshev-Gauss-Lobatto (CGL) points on the interval $[a, b]$ defined by

$$
a=x_{0}<x_{1}<\cdots<x_{N+1}=b, \quad x_{j}=\frac{a+b}{2}+\frac{b-a}{2} \cos \left(\frac{j \pi}{N+1}\right) .
$$

For a time $t>0$, let $\mathbf{u}(t)=\left[u_{0}(t), \cdots, u_{N+1}(t)\right]^{T}$, where $u_{j}(t):=u\left(t, x_{j}\right)$, $j=0,1, \cdots, N+1$, and let $\widehat{\mathbf{D}}$ be the first-order $(N+2) \times(N+2)$ Chebyshev differentiation matrix associated with the CGL points $(2.2)$ (see $[9,15]$ ). Then the pseudospectral approximations for the partial derivatives $\frac{\partial}{\partial x} u\left(t, x_{j}\right), \frac{\partial^{2}}{\partial x^{2}} u\left(t, x_{j}\right), \cdots$ can be stated as

$$
\frac{\partial}{\partial x} u\left(t, x_{j}\right)=(\widehat{\mathbf{D}} \mathbf{u}(t))_{j}, \quad \frac{\partial^{2}}{\partial x^{2}} u\left(t, x_{j}\right)=\left(\widehat{\mathbf{D}}^{2} \mathbf{u}(t)\right)_{j}, \cdots \quad 1 \leq j \leq N,
$$

where $(\mathbf{a})_{j}$ denotes the $j^{\text {th }}$ component of the vector $\mathbf{a}$. by

Define the internal vector-valued function $\tilde{\mathbf{u}}(t)$ using the internal nodes $\left\{x_{j}\right\}_{j=1}^{N}$

$$
\tilde{\mathbf{u}}(t)=\left[u\left(t, x_{1}\right), \cdots, u\left(t, x_{N}\right)\right]^{T}
$$

and write the vector $\mathbf{u}(t)$ using the boundary conditions in (2.1) and the boundaryinternal nodes $\left\{x_{j}\right\}_{j=0}^{N+1}$ as

$$
\mathbf{u}(t)=\left[q_{1}(t), u\left(t, x_{1}\right), \cdots, u\left(t, x_{N}\right), q_{2}(t)\right]^{T} .
$$

For convenience, let the notation $\lfloor\lfloor\mathbf{a}\rceil\rceil$ denote the vector removed the first and last components of the column vector a and let $X \odot Y$ mean the Hadamard product of two vectors $X$ and $Y$. Using these notations, we have the pseudo-spectral discretizations for spatial derivatives of (2.1) and it becomes a nonlinear system of ODE's involving time variable only

$$
\left\{\begin{array}{l}
\frac{\partial}{\partial t} \tilde{\mathbf{u}}(t)=\mathrm{F}(t, \tilde{\mathbf{u}}(t)), \quad 0<t \leq t_{\text {end }} \\
\tilde{\mathbf{u}}(0)=\left[Q\left(x_{1}\right), \cdots, Q\left(x_{N}\right)\right]^{T}
\end{array}\right.
$$

where

$$
\mathrm{F}(t, \tilde{\mathbf{u}}(t)):=\epsilon\left\lfloor\widehat{\mathbf{D}}^{2} \mathbf{u}\right\rceil-\tilde{\mathbf{u}} \odot\lfloor\widehat{\mathbf{D}} \mathbf{u}\rceil .
$$

Hence, we are now in a position to apply ECEM developed in [22] for time discretizations. For this purpose, consider a general nonlinear stiff system of ODEs instead of (2.4):

$$
\frac{d \Phi}{d t}=\mathrm{F}(t, \Phi(t)), \quad t \in\left(t_{0}, t_{\text {end }}\right] ; \quad \Phi\left(t_{0}\right)=\Phi_{0},
$$

where $\Phi(t)=\left[\phi_{1}(t), \cdots, \phi_{M}(t)\right]^{T}, \mathrm{~F}(t, \Phi(t))=\left[f_{1}(t, \Phi(t)), \cdots, f_{M}(t, \Phi(t))\right]^{T}$ and $\Phi_{0}$ is a given initial data and $\mathrm{F}(t, \Phi(t))$ satisfies all the necessary requirement for 
the existence of the unique solution. Here, the notation $T$ denotes the transpose of a vector.

\subsection{Stiffness}

Before going further to introduce ECEM for (2.5), we investigate first of all how the stiffness of the discrete system (2.4) in space is affected by the relation between the viscosity $\epsilon$ and the number $M:=N$ of interior points. The stiffness for the system is commonly measured by the ratio (called stiffness ratio) (see [31] for example) $\frac{\max |\operatorname{Re}(\lambda)|}{\min |\operatorname{Re}(\lambda)|}$, where $\lambda$ are the eigenvalues of the gradient matrix $\nabla \mathrm{F}(t, \tilde{\mathbf{u}}(t))$ in $(2.4)$ and $\operatorname{Re}(z)$ denotes the real part of the complex number $z$. To see the stiffness, we consider the system (2.4) obtained from the Burgers' problem (2.1) on the interval $[-1,1]$ with the initial and boundary conditions

$$
Q(x)=-\sin (\pi x), \quad q_{i}(t)=0, i=1,2,
$$

respectively. The stiffness ratios are calculated with two ways and listed in Table 1 and 2. Table 1 shows the stiffness ratio for the viscosity $\epsilon=1,10^{-1}, 10^{-2}, 10^{-3}$ and the time $0.03 \leq t \leq 0.18$ when the fixed number $M=255$ of interior points are chosen, while the results in Table 2 are obtained by increasing the number $M$ of interior points from 31 to 127 with the fixed viscosity $\epsilon=1$. The results in Table 1 and 2 show that the stiffness is fairly depending on both the viscosity $\epsilon$ and the number $M$ of interior points. From these results in Table 1 and 2, the problem (2.4) becomes a highly stiff system when the viscosity $\epsilon$ is sufficiently small as other numerical methods reveal (see [31]).

\section{Time discretizations}

Assume that the time domain $\left[t_{0}, t_{\text {end }}\right]$ is divided with the uniform time length $\tau=\left(t_{\text {end }}-t_{0}\right) / \mathfrak{N}$ such that

$$
t_{0}<t_{1}<\cdots<t_{\mathfrak{N}}=t_{\text {end }}, \quad t_{j}=t_{0}+j \tau,
$$

where $\mathfrak{N}$ is a given positive integer. Assume that an approximation $\mathrm{Y}_{n}$ of $\Phi(t)$ at time $t=t_{n}$ is given. Consider the Euler polygon defined by

$$
\mathrm{Y}(t)=\mathrm{Y}_{n}+\left(t-t_{n}\right) \mathrm{F}\left(t_{n}, \mathrm{Y}_{n}\right), \quad t \in\left[t_{n}, t_{n+1}\right] .
$$

By (2.5) and (3.1), the perturbation $\Psi(t)=\left[\psi_{1}(t), \cdots, \psi_{M}(t)\right]^{T}$ on $\left[t_{n}, t_{n+1}\right]$ given by

$$
\Psi(t)=\Phi(t)-\mathrm{Y}(t)
$$

satisfies the following ODE:

(3.3) $\frac{d}{d t} \Psi(t)=\nabla \mathrm{F}(t, \mathrm{Y}(t)) \Psi(t)+\mathrm{G}(t)+\frac{1}{2} \Psi(t)^{T} \nabla^{2} \mathrm{~F}(t, \Xi(t)) \Psi(t)^{T}, t \in\left(t_{n}, t_{n+1}\right)$, 
Table 1: The stiffness ratio of the nonlinear system of ODEs (2.4) with the fixed number $M=255$ of interior points

\begin{tabular}{c|cccccc}
\hline$\epsilon$ & 0.03 & 0.06 & 0.09 & 0.12 & 0.15 & 0.18 \\
\hline $1 . \mathrm{e}-3$ & $3.68 \mathrm{e}+10$ & $8.29 \mathrm{e}+09$ & $6.44 \mathrm{e}+10$ & $3.58 \mathrm{e}+10$ & $1.94 \mathrm{e}+10$ & $6.23 \mathrm{e}+10$ \\
$1 . \mathrm{e}-2$ & $1.38 \mathrm{e}+11$ & $3.48 \mathrm{e}+11$ & $7.86 \mathrm{e}+12$ & $1.07 \mathrm{e}+11$ & $2.75 \mathrm{e}+11$ & $7.01 \mathrm{e}+11$ \\
$1 . \mathrm{e}-1$ & $6.47 \mathrm{e}+10$ & $5.85 \mathrm{e}+09$ & $4.59 \mathrm{e}+09$ & $4.41 \mathrm{e}+09$ & $3.50 \mathrm{e}+09$ & $2.83 \mathrm{e}+09$ \\
$1 . \mathrm{e}-0$ & $1.13 \mathrm{e}+08$ & $1.04 \mathrm{e}+08$ & $9.80 \mathrm{e}+07$ & $9.33 \mathrm{e}+07$ & $9.06 \mathrm{e}+07$ & $8.84 \mathrm{e}+07$ \\
\hline
\end{tabular}

Table 2: The stiffness ratio of the nonlinear system of ODEs (2.4) with the fixed viscosity $\epsilon=1$

\begin{tabular}{c|cccccc}
\hline$M$ & 0.03 & 0.06 & 0.09 & 0.12 & 0.15 & 0.18 \\
\hline 31 & $2.78 \mathrm{e}+04$ & $2.55 \mathrm{e}+04$ & $2.40 \mathrm{e}+04$ & $2.30 \mathrm{e}+04$ & $2.22 \mathrm{e}+04$ & $2.17 \mathrm{e}+04$ \\
63 & $4.42 \mathrm{e}+05$ & $4.07 \mathrm{e}+05$ & $3.83 \mathrm{e}+05$ & $3.66 \mathrm{e}+05$ & $3.54 \mathrm{e}+05$ & $3.46 \mathrm{e}+05$ \\
127 & $7.07 \mathrm{e}+06$ & $6.51 \mathrm{e}+06$ & $6.12 \mathrm{e}+06$ & $5.85 \mathrm{e}+06$ & $5.66 \mathrm{e}+06$ & $5.53 \mathrm{e}+06$ \\
\hline
\end{tabular}

where $\Xi(t)$ is a function between $\mathrm{Y}(t)$ and $\Phi(t), \nabla \mathrm{F}(t, \mathrm{Y}(t))$ and $\nabla^{2} \mathrm{~F}(t, \mathrm{Y}(t))$ are the gradient and Hessian matrices of $\mathrm{F}$, respectively and the vector $\mathrm{G}(t)=$ $\left[g_{1}(t), g_{2}(t), \cdots, g_{M}(t)\right]^{T}$ is given by

$$
\mathrm{G}(t)=\mathrm{F}(t, \mathrm{Y}(t))-\mathrm{F}\left(t_{n}, \mathrm{Y}_{n}\right) .
$$

By the change of variable $t=t_{s}=t_{n}+\frac{\tau}{2}(1+s)$ from the computational region $\left[t_{n}, t_{n+1}\right]$ to the reference domain $[-1,1]$, one may have a system of asymptotically first-order linear ODE's in the reference domain $[-1,1]$ instead of $(3.3)$ as follows:

$$
\frac{d}{d s} \bar{\Psi}(s)=\frac{\tau}{2}\left(\nabla \mathrm{F}\left(t_{s}, \mathrm{Y}\left(t_{s}\right)\right) \bar{\Psi}(s)+\mathrm{G}\left(t_{s}\right)\right)+O\left(\tau\|\bar{\Psi}(s)\|^{2}\right), \quad s \in[-1,1],
$$

where $\bar{\Psi}(s):=\left[\bar{\psi}_{1}(s), \cdots, \bar{\psi}_{M}(s)\right]^{T}$ with $\bar{\psi}_{i}(s):=\psi_{i}(t)=\psi_{i}\left(t_{s}\right)$ and $\|\cdot\|$ is a vector norm.

We note that one way to avoid the calculation of the gradient matrix $\nabla \mathrm{F}(t, \mathrm{Y}(t))$ in (3.3) is to replace each component of $\nabla \mathrm{F}(t, \mathrm{Y}(t))$ with its forward difference quotient

$$
\frac{\partial f_{i}}{\partial y_{j}}(t, \mathrm{Y}(t)) \approx \frac{f_{i}\left(t, \mathrm{Y}(t)+\lambda \mathbf{e}_{j}\right)-f_{i}(t, \mathrm{Y}(t))}{\lambda}, \quad i, j=1, \cdots, M,
$$

where $\mathbf{e}_{j}$ is the $j^{\text {th }}$ column of the identity matrix of order $M$ and $\lambda$ is a sufficiently small positive number. In such a replacement, the asymptotic part in (3.5) will become $O\left(\tau\|\bar{\Psi}(s)\|^{2}+\lambda\|\bar{\Psi}(s)\|\right)$. Then, in any case using either the gradient or its 
approximation by forward difference scheme, we can rewrite the asymptotic system (3.5) in one form without the error term as follows:

$$
\frac{d}{d s} \bar{\Psi}(s)=\frac{\tau}{2}\left(K(s) \bar{\Psi}(s)+\mathrm{G}\left(t_{s}\right)\right),
$$

where the matrix $K(s):=\left(\varphi_{i j}(s)\right)$ is defined by either

$$
\varphi_{i j}(s)=\frac{\partial}{\partial y_{j}} f_{i}\left(t_{s}, \mathrm{Y}\left(t_{s}\right)\right),
$$

or

$$
\varphi_{i j}(s)=\frac{f_{i}\left(t, \mathrm{Y}(t)+\lambda \mathbf{e}_{j}\right)-f_{i}(t, \mathrm{Y}(t))}{\lambda} .
$$

Remark 3.1. Recalling that the Euler method has the local truncation error $O\left(\tau^{2}\right)$, one can guess the deleted error term in (3.6) may be quite small and can be ignored. In fact, one may prove that the error is $O\left(\tau^{5}\right)$ provided $\lambda=O\left(\tau^{2}\right)$ (see [22]). It is remarkable that once the error term is disregarded, the system (3.6) will be completely linear and its approximation scheme becomes an explicit type.

For an approximation of (3.6), the Chebyshev-collocation method (CCM) will be used because it is known that CCM have a good stability for the stiff system (2.5) (see $[22,29]$ ). Let $\left\{s_{j}:=-\cos \frac{\pi j}{4}\right\}_{j=0}^{4}$ be the CGL points in $[-1,1]$ and let $\left\{l_{k}(s)\right\}_{k=0}^{4}$ be the fourth-order interpolation polynomials defined by

$$
l_{k}(s)=\frac{\alpha_{k}}{4} \sum_{j=0}^{4} T_{j}^{\prime \prime}\left(s_{k}\right) T_{j}(s), \quad \alpha_{k}= \begin{cases}1, & k=0,4, \\ 2, & \text { otherwise }\end{cases}
$$

where $T_{j}(s)=\cos \left(j \cos ^{-1} s\right)$ is the first kind Chebyshev polynomial of degree $j$ and the double prime indicates that both the first and last terms in the summation are to be halved. Then, each component $\bar{\psi}_{i}(s)$ of the solution $\bar{\Psi}(s)$ of $(3.6)$ can be approximated by

$$
\bar{\psi}_{i}(s) \approx \sum_{k=0}^{4} \bar{\psi}_{i}\left(s_{k}\right) l_{k}(s), \quad i=1, \cdots, M,
$$

whose error has the asymptotic behavior $O\left(\tau^{5}\right)$ if $\psi_{i}(t)$ is fifth-times continuously differential function [22]. By substituting (3.8) into (3.6), one may approximate the equations (3.6) with

$$
\begin{aligned}
\sum_{k=1}^{4}\left(\bar{\psi}_{i}\left(s_{k}\right) i_{k}(s)-\frac{\tau}{2} \sum_{j=1}^{M} \varphi_{i j}(s) \bar{\psi}_{j}\left(s_{k}\right) l_{k}(s)\right) & =\frac{\tau}{2}\left(\sum_{j=1}^{M} \varphi_{i j}(s) \bar{\psi}_{j}\left(s_{0}\right) l_{0}(s)+g_{i}\left(t_{s}\right)\right) \\
& -\bar{\psi}_{i}\left(s_{0}\right) \dot{l}_{0}(s), \quad i=1, \cdots, M,
\end{aligned}
$$


where $i_{k}(s)$ denotes the derivative of $l_{k}(s)$. To determine $4 M$ unknown coefficients $\bar{\psi}_{i}\left(s_{k}\right), i=1, \cdots, M, k=1, \cdots, 4$, we collocate the equations (3.9) at the points $s_{\nu}, \nu=1, \cdots, 4$. Then, by the facts $l_{k}\left(s_{\nu}\right)=\delta_{k \nu}$, where $\delta_{k \nu}$ denotes the Kronecker delta function, one may have the discrete system

$$
\sum_{k=1}^{4} \bar{\psi}_{i}\left(s_{k}\right) \dot{l}_{k}\left(s_{\nu}\right)-\frac{\tau}{2} \sum_{j=1}^{M} \varphi_{i j}\left(s_{\nu}\right) \bar{\psi}_{j}\left(s_{\nu}\right)=\frac{\tau}{2} g_{i}\left(t_{s_{\nu}}\right)-\bar{\psi}_{i}\left(s_{0}\right) \dot{l}_{0}\left(s_{\nu}\right),
$$

where $i=1, \cdots, M, \quad \nu=1, \cdots, 4$. Note that $\bar{\psi}_{i}\left(s_{0}\right)$ is the $i^{\text {th }}$ component of the perturbation $\Phi\left(t_{n}\right)-\mathrm{Y}_{n}$, which are also unknown. However, they are quite small and can be neglected provided $Y_{n}$ is an accurate approximation of $\Phi\left(t_{n}\right)$. Hence, truncating the last term $\bar{\psi}_{i}\left(s_{0}\right) \dot{l}_{0}\left(s_{\nu}\right)$ in (3.10) gives a fully discrete linear system as follows: for $i=1, \cdots, M, \nu=1, \cdots, 4$,

$$
\sum_{k=1}^{4} \bar{\psi}_{i}\left(s_{k}\right) \dot{i}_{k}\left(s_{\nu}\right)-\frac{\tau}{2} \sum_{j=1}^{M} \varphi_{i j}\left(s_{\nu}\right) \bar{\psi}_{j}\left(s_{\nu}\right)=\frac{\tau}{2} g_{i}\left(t_{s_{\nu}}\right),
$$

with $4 M$ equations and $4 M$ unknowns $\bar{\psi}_{i}\left(s_{k}\right)$. In order to rewrite (3.11) in a matrix form, for $1 \leq j, k \leq 4$, let

$$
\mathrm{L}=\left(L_{j k}:=i_{k}\left(s_{j}\right)\right)_{4 \times 4}, \quad \mathrm{~J}^{(\mu, \nu)}=\left(J_{j k}^{(\mu, \nu)}:=\varphi_{\mu \nu}\left(s_{j}\right) \delta_{j k}\right)_{4 \times 4}, \quad 1 \leq \mu, \nu \leq M
$$

and

$$
\begin{aligned}
& \mathbf{c}=\left[\bar{\psi}_{1}\left(s_{1}\right) \cdots \bar{\psi}_{1}\left(s_{4}\right), \bar{\psi}_{2}\left(s_{1}\right) \cdots \bar{\psi}_{M}\left(s_{4}\right)\right]^{T}, \\
& \mathbf{g}=\left[g_{1}\left(t_{s_{1}}\right), \cdots, g_{1}\left(t_{s_{4}}\right), g_{2}\left(t_{s_{1}}\right), \cdots, g_{M}\left(t_{s_{4}}\right)\right]^{T} .
\end{aligned}
$$

Using $\mathbf{L}$ and $J^{(\mu, \nu)}$ in (3.12), we define matrices $\hat{L}$ and $\widehat{J}$ by

$$
\widehat{\mathbf{L}}=\mathbf{I} \otimes \mathbf{L} \quad \text { and } \quad \widehat{\jmath}=\left[\begin{array}{cccc}
\mathrm{J}^{(1,1)} & \mathrm{J}^{(1,2)} & \ldots & \mathrm{J}^{(1, M)} \\
\mathrm{J}^{(2,1)} & \mathrm{J}^{(2,2)} & \ldots & \mathrm{J}^{(2, M)} \\
\vdots & \vdots & \ddots & \vdots \\
\mathrm{J}^{(M, 1)} & \mathrm{J}^{(M, 2)} & \cdots & \mathrm{J}^{(M, M)}
\end{array}\right]
$$

where $\mathbf{I} \otimes \mathbf{L}$ denotes the tensor product of $\mathbf{I}$ and $\mathbf{L}$ and $\mathbf{I}$ denotes an $M \times M$ identity matrix.

Lemma 3.1. In matrix terminology, (3.11) can be expressed as

$$
\left(\widehat{L}-\frac{\tau}{2} \widehat{J}\right) \mathbf{c}=\frac{\tau}{2} \mathbf{g} .
$$

Moreover, for a sufficiently small $\tau$, it has a unique solution if the gradient matrix $\nabla \mathrm{F}(t, \Phi(t))$ is uniformly bounded. 
Proof. The representation comes from a straightforward calculation. Note that the matrix $\widehat{J}$ is uniformly bounded whenever the gradient matrix $\nabla \mathrm{F}(t, \Phi(t))$ is uniformly bounded and the invertibility of $\mathbf{L}$ (see [22]) leads to the invertibility of $\hat{\mathrm{L}}$. Hence for sufficiently small $\tau$ the linear system (3.14) has a unique solution.

Note that each $\bar{\psi}_{i}\left(s_{4}\right)$ is an approximation of the $i^{\text {th }}$ component of the vector $\Phi\left(t_{n+1}\right)-\mathrm{Y}\left(t_{n+1}\right)$. Thus if we define $\mathbf{c}^{(4)}:=\left[\bar{\psi}_{1}\left(s_{4}\right), \bar{\psi}_{2}\left(s_{4}\right), \cdots, \bar{\psi}_{M}\left(s_{4}\right)\right]^{T}$ from the solution $\mathbf{c}$ of (3.14), then one can see

$$
\Phi\left(t_{n+1}\right) \approx \mathrm{Y}\left(t_{n+1}\right)+\mathbf{c}^{(4)}=\mathrm{Y}_{n}+\tau \mathrm{F}\left(t_{n}, \mathrm{Y}_{n}\right)+\mathbf{c}^{(4)} .
$$

Hence it is naturally to define the approximation $Y_{n+1}$ of $\Phi\left(t_{n+1}\right)$ as follows:

$$
\left\{\begin{aligned}
\mathrm{Y}_{n+1} & =\mathrm{Y}_{n}+\tau \mathrm{F}\left(t_{n}, \mathrm{Y}_{n}\right)+\mathbf{c}^{(4)}, \quad n \geq 0 \\
\mathrm{Y}_{0} & =\Phi_{0} .
\end{aligned}\right.
$$

Remark that the scheme (3.15) is identically same with the Euler method if we disregard the term $\mathbf{c}^{(4)}$ in the recurrence relation (3.15). The term $\mathbf{c}^{(4)}$ serves as a correction for the error in the Euler method. In this view, we prefer to say the approximation scheme (3.15) as an error corrected Euler method (ECEM).

Theorem 3.2. The scheme (3.15) has a local truncation error $O\left(\tau^{5}\right)$ and hence has a fourth-order convergence rate.

Proof. First note that the error term has the behavior $O\left(\tau^{5}\right)$ (see Remark 3.1) and truncation error for the fourth-order Chebyshev interpolation polynomial has $O\left(\tau^{5}\right)$ (see (3.8)). Then, following the proof in [22] for the scalar stiff problem line by line, one may have the conclusion.

\section{Numerical experiments}

In this section, throughout several numerical experiments of Burgers' equations we will provide numerical evidences on the effectiveness and accuracy of the developed ECEM. These aims will be done by comparing ECEM to other numerical approaches based on the finite difference method [16, 23], finite element method [28], cubic spline and sinc-function methods [31, 32], and the fourth-order Runge-Kutta method [21] (RK4) and the BDF-type Chebyshev approximation based fourth-order implicit Runge-Kutta method [29] (CCM). The numerical errors are measured by the following maximum and $l_{2}$ norms

$$
E_{1}(t, \tau):=\max _{1 \leq j \leq M}\left|u\left(t, x_{j}\right)-u_{j}(t)\right|, E_{2}(t, \tau):=\left(\sum_{j=1}^{M}\left(u\left(t, x_{j}\right)-u_{j}(t)\right)^{2}\right)^{1 / 2},
$$

where $u\left(t, x_{j}\right)$ represents the exact solution to $(2.1)$ and $u_{j}(t)$ represents its approximation at space $x_{j}$ and time $t$. The convergence rate will be measured by

$$
\text { rate }:=\frac{\log \left(E_{i}\left(t, \tau_{1}\right) /\left(E_{i}\left(t, \tau_{2}\right)\right)\right.}{\log \left(\tau_{1} / \tau_{2}\right)}, \quad i=1,2,
$$


where $\tau_{1}$ and $\tau_{2}$ are time lengths.

Before going further numerical demonstrations, it will be explained the reason why we prefer the integral form in [4] to an infinite series form in [31] for the exact solution given in the following example.

Example 4.1. Consider the Burgers' problem (2.1) with the initial and boundary conditions in (2.6).

The solution of the Burgers equation of Example 4.1 can be written as either

$$
u(t, x)=-\frac{\int_{-\infty}^{+\infty} \sin (\pi(x-\sqrt{4 \epsilon t} \xi)) \exp \left(\frac{-\cos (\pi(x-\sqrt{4 \epsilon t} \xi))}{2 \epsilon \pi}\right) \exp \left(-\xi^{2}\right) d \xi}{\int_{-\infty}^{+\infty} \exp \left(\frac{-\cos (\pi(x-\sqrt{4 \epsilon t} \xi))}{2 \epsilon \pi}\right) \exp \left(-\xi^{2}\right) d \xi},
$$

where $a_{n}=(-1)^{n} I_{n}(1 /(2 \pi \epsilon))$ and $I_{n}(z)$ denotes the modified Bessel function of the first kind or

$$
u(t, x)=\frac{4 \pi \epsilon \sum_{n=1}^{\infty} n a_{n} \exp \left(-n^{2} \pi^{2} \epsilon t\right) \sin (n \pi x)}{a_{0}+2 \sum_{n=1}^{\infty} a_{n} \exp \left(-n^{2} \pi^{2} \epsilon t\right) \cos (n \pi x)} .
$$

The numerical calculation will be done by the Gauss-Hermite integration with 200 nodes for the integral form (4.1) and the buildin function besseli in Matlab and by the 200 finite sum for the approximation of the infinite series form (4.2). According to the numerical results in Fig. 1 with two viscosities $\epsilon=0.01$ and $\epsilon=0.003$, both expressions (4.1) and (4.2) are very good for the relatively large viscosity $\epsilon=0.01$ but for the small viscosity $\epsilon=0.003$ the integral form (4.1) is better than the series form (4.2). Hence, we will use the integral form (4.1) for the numerical comparisons.

To show the effectiveness of ECEM, we will compare it with two time stepping approaches CCM [29] and RK4 [21], where both methods used the same spatial discretization with ECEM, while CCM [29] and RK4 [21] used a fourth-order implicit type Runge-Kutta method based on BDF-type Chebyshev approximation and the fourth-order explicit method, respectively for the time discretization. The numerical approximations by RK4 with viscosity $\epsilon=0.01$ are reported in Table 3 at time $t=0.3$ when $M=31$ or $M=63$, where $\infty$ means the undefined numerical result. The results show that RK4 gives unsatisfactory results because of the stiffness for the problem. In particular, the time length $\tau \leq 3 \times 10^{-7}$ must be chosen to get a numerical solution within the maximum error $E_{1}(0.3, \tau)=1.51 \times 10^{-9}$ when $M=255$ and $\epsilon=0.01$. It means RK4 requires much more computational costs than ECEM does (see Table 4).

For the discussions on numerical computations shown in Table 4 and 5 , we will take $M=511$ for $\epsilon=0.003$ and $M=255$ for $\epsilon=0.01$. In both cases, the time 
Table 3: The errors and convergence rates by RK4 with different time length $\tau$ at $t=0.3$ for Example 4.1 with the viscosity $\epsilon=0.01$.

\begin{tabular}{|c|c|c|c|c|c|c|c|c|c|c|}
\hline & \multicolumn{5}{|c|}{$\mathrm{M}=31[21]$} & \multicolumn{5}{|c|}{$\mathrm{M}=63[21]$} \\
\hline$\tau$ & $E_{1}(t, \tau)$ & rate & $E_{2}(t, \tau)$ & rate & feval & $E_{1}(t, \tau)$ & rate & $E_{2}(t, \tau)$ & rate & feval \\
\hline$\frac{0.3}{10}$ & $\infty$ & & $\infty$ & & 40 & $\infty$ & & $\infty$ & & 40 \\
\hline$\frac{0.3}{20}$ & $\infty$ & & $\infty$ & & 80 & $\infty$ & & $\infty$ & & 80 \\
\hline$\frac{0.3}{40}$ & $8.00 \mathrm{e}-1$ & & $1.32 \mathrm{e}-0$ & & 160 & $\infty$ & & $\infty$ & & 160 \\
\hline$\frac{0.3}{80}$ & $2.61 \mathrm{e}-2$ & 4.94 & $4.69 \mathrm{e}-2$ & 4.81 & 320 & $\infty$ & & $\infty$ & & 320 \\
\hline
\end{tabular}

Table 4: Comparisons between ECEM and CCM [29] at $t=0.3$ for Example 4.1 with $\epsilon=0.01, M=255$.

\begin{tabular}{cccccc|ccccc}
\hline \multicolumn{5}{c|}{ ECEM } & \multicolumn{5}{c}{ CCM $[29]$} \\
\hline$\tau$ & $E_{1}(t, \tau)$ & rate & $E_{2}(t, \tau)$ & rate & feval & $E_{1}(t, \tau)$ & rate & $E_{2}(t, \tau)$ & rate & feval \\
\hline$\frac{0.3}{10}$ & $7.86 \mathrm{e}-6$ & & $2.80 \mathrm{e}-5$ & & 30 & $6.09 \mathrm{e}-7$ & & $1.96 \mathrm{e}-6$ & & 70 \\
$\frac{0.3}{20}$ & $4.32 \mathrm{e}-7$ & 4.19 & $1.59 \mathrm{e}-6$ & 4.14 & 60 & $4.12 \mathrm{e}-8$ & 3.89 & $1.31 \mathrm{e}-7$ & 3.90 & 128 \\
$\frac{0.3}{40}$ & $2.48 \mathrm{e}-8$ & 4.12 & $9.54 \mathrm{e}-8$ & 4.06 & 120 & $4.11 \mathrm{e}-9$ & 3.33 & $1.08 \mathrm{e}-8$ & 3.60 & 244 \\
$\frac{0.3}{80}$ & $2.80 \mathrm{e}-9$ & 3.14 & $8.89 \mathrm{e}-9$ & 3.42 & 240 & $1.64 \mathrm{e}-9$ & 1.33 & $6.76 \mathrm{e}-9$ & 0.68 & 466 \\
\hline
\end{tabular}

Table 5: Comparisons between ECEM and CCM [29] at $t=0.3$ for Example 4.1 for $\epsilon=0.003, M=511$.

\begin{tabular}{cccccc|ccccc}
\hline \multicolumn{5}{c|}{ ECEM } & \multicolumn{5}{c}{ CCM $[29]$} \\
\hline$\tau$ & $E_{1}(t, \tau)$ & rate & $E_{2}(t, \tau)$ & rate & feval & $E_{1}(t, \tau)$ & rate & $E_{2}(t, \tau)$ & rate & feval \\
\hline$\frac{0.3}{10}$ & $1.41 \mathrm{e}-4$ & & $3.88 \mathrm{e}-4$ & & 30 & $4.16 \mathrm{e}-6$ & & $1.18 \mathrm{e}-5$ & & 70 \\
$\frac{0.3}{20}$ & $9.25 \mathrm{e}-6$ & 3.93 & $2.35 \mathrm{e}-5$ & 4.05 & 60 & $3.49 \mathrm{e}-7$ & 3.58 & $9.24 \mathrm{e}-7$ & 3.67 & 130 \\
$\frac{0.3}{40}$ & $5.59 \mathrm{e}-7$ & 4.05 & $1.38 \mathrm{e}-6$ & 4.09 & 120 & $2.30 \mathrm{e}-8$ & 3.92 & $6.77 \mathrm{e}-8$ & 3.77 & 250 \\
$\frac{0.3}{80}$ & $3.42 \mathrm{e}-8$ & 4.03 & $8.47 \mathrm{e}-8$ & 4.03 & 240 & $5.06 \mathrm{e}-9$ & 2.18 & $1.94 \mathrm{e}-8$ & 1.80 & 484 \\
\hline
\end{tabular}



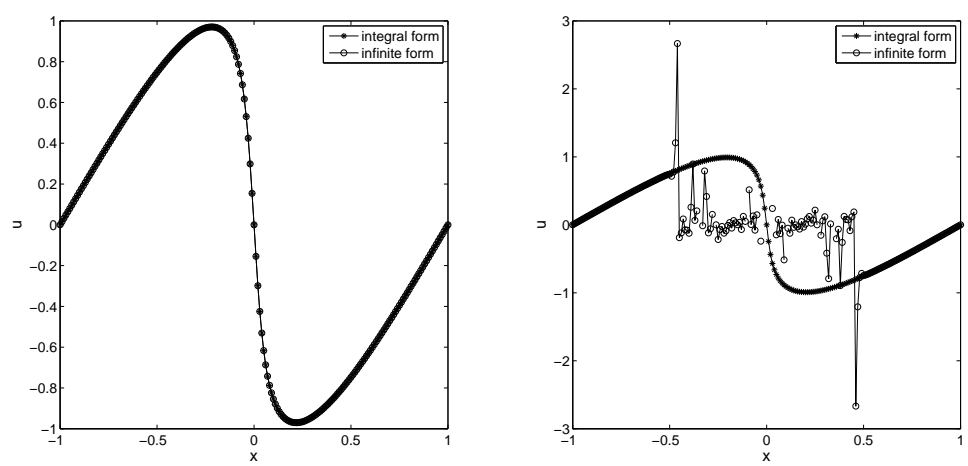

Figure 1: Comparison of two expressions of the exact solution for Example 4.1 , at time $t=0.3$ with fixed $\epsilon=0.01$ (left) and $\epsilon=0.003$ (right), respectively.

length is given by $\tau=\frac{0.3}{2^{k} \times 10}, k=0,1,2,3$. The column feval denotes the number of function evaluations for the vector function $\mathrm{F}(t, \mathrm{Y}(t))$. The stopping criterion for the Newton iteration needed in CCM [29] is taken as the maximum norm of residual less than $\tau^{4}$ to get the fourth-order convergence. The numerical results show that ECEM has a convergence order 4 and the behaviors of errors $E_{i}(t, \tau), i=1,2$ for both ECEM and CCM look like similar. However, ECEM reduces about one-half of computational costs in the sense of function evaluations (feval) comparing to CCM.

Also, the results of Table 4 show that ECEM is more efficient than LRAM [31], where for the spatial and time discretization, LRAM used the differential quadrature method using sinc-function and the linearized and rational Pade approximation for the matrix exponential, respectively, which require a similar computational costs with the present method for the same number of spatial points. In fact, to get the numerical approximation having the maximum error $4.43 e-4$ at time $t=0.3$ with $\epsilon=0.01$, LRAM is required the time length $\tau=0.003$ and the number $M=500$ of interior points, which is costly compared with ECEM.

Fig. 2 shows the numerical solutions at different times for $\epsilon=10^{-2}, 3 \times 10^{-3}$. For the viscosity $\epsilon=0.01$ we take $M=127$ and $\tau=0.1$ and we take $M=255$ and $\tau=0.01$ for the viscosity $\epsilon=0.003$. The numerical solutions with small viscosities ranged from $\epsilon=10^{-3}$ to $\epsilon=10^{-8}$ for $M=127$ and $\tau=0.1$ are provided in Fig. 3.

Example 4.2. Consider Burgers' equation (2.1) with the initial condition $Q(x)=$ $4 x(1-x)$ over domain $[0,1]$ and zero boundary conditions $q_{i}(t)=0, i=1,2$. The viscosity constants are chosen either $\epsilon=1$ or $\epsilon=0.01$.

For the case $\epsilon=1$, ECEM is compared to the known numerical results obtained from the finite difference and element methods ([16] and [28]) in Table 6. For discussions on three methods in the sense of time step $\tau$ and number of interior 
Table 6: Comparisons of Example 4.2 at different times for $\epsilon=1$.

\begin{tabular}{cc|cc|c|c}
\hline$x$ & $t$ & Hassanien [16] & Öziş [28] & ECEM+PS & Exact Solution \\
\hline & & \multicolumn{2}{|c|}{$M=79$} & $M=11$ & \\
\hline \multirow{2}{*}{0.25} & 0.10 & 0.26148 & 0.26245 & 0.26148 & 0.26148 \\
& 0.15 & 0.16148 & 0.16157 & 0.16148 & 0.16148 \\
& 0.20 & 0.09947 & 0.09948 & 0.09947 & 0.09947 \\
& 0.25 & 0.06109 & 0.06111 & 0.06109 & 0.06108 \\
\hline 0.50 & 0.10 & 0.38342 & 0.38314 & 0.38342 & 0.38342 \\
& 0.15 & 0.23405 & 0.23394 & 0.23406 & 0.23406 \\
& 0.20 & 0.14289 & 0.14287 & 0.14289 & 0.14289 \\
& 0.25 & 0.08723 & 0.08729 & 0.08723 & 0.08723 \\
\hline 0.75 & 0.10 & 0.28157 & 0.28004 & 0.28157 & 0.28157 \\
& 0.15 & 0.16974 & 0.16948 & 0.16974 & 0.16974 \\
& 0.20 & 0.10265 & 0.10261 & 0.10266 & 0.10266 \\
& 0.25 & 0.06229 & 0.06230 & 0.06229 & 0.06229 \\
\hline
\end{tabular}

Table 7: Comparisons of Example 4.2 at different times for $\epsilon=0.01$.

\begin{tabular}{|c|c|c|c|c|c|}
\hline \multirow[t]{3}{*}{$x$} & \multirow[t]{3}{*}{$t$} & Kutluay[23] & Kutluay [23] & \multirow{2}{*}{$\begin{array}{c}\mathrm{ECEM}+\mathrm{PS} \\
M=36\end{array}$} & \multirow[t]{2}{*}{ Exact Solution } \\
\hline & & \multicolumn{2}{|c|}{$M=79$} & & \\
\hline & & $\tau=0.001$ & $\tau=0.001$ & $\tau=0.01$ & \\
\hline \multirow[t]{5}{*}{0.25} & 0.4 & 0.36296 & 0.36185 & 0.36226 & 0.36226 \\
\hline & 0.6 & 0.28217 & 0.28193 & 0.28204 & 0.28204 \\
\hline & 0.8 & 0.23043 & 0.23046 & 0.23045 & 0.23045 \\
\hline & 1.0 & 0.19463 & 0.19474 & 0.19469 & 0.19469 \\
\hline & 3.0 & 0.07611 & 0.07617 & 0.07613 & 0.07613 \\
\hline \multirow[t]{5}{*}{0.50} & 0.4 & 0.69591 & 0.67851 & 0.68368 & 0.68368 \\
\hline & 0.6 & 0.55351 & 0.54508 & 0.54831 & 0.54832 \\
\hline & 0.8 & 0.45625 & 0.45176 & 0.45371 & 0.45371 \\
\hline & 1.0 & 0.38705 & 0.38446 & 0.38568 & 0.38568 \\
\hline & 3.0 & 0.15220 & 0.15215 & 0.15218 & 0.15218 \\
\hline \multirow[t]{5}{*}{0.75} & 0.4 & 0.95925 & 0.91169 & 0.92050 & 0.92050 \\
\hline & 0.6 & 0.80197 & 0.77402 & 0.78298 & 0.78299 \\
\hline & 0.8 & 0.67267 & 0.56617 & 0.66272 & 0.66272 \\
\hline & 1.0 & 0.57501 & 0.56478 & 0.56932 & 0.56932 \\
\hline & 3.0 & 0.22796 & 0.22746 & 0.22774 & 0.22774 \\
\hline
\end{tabular}



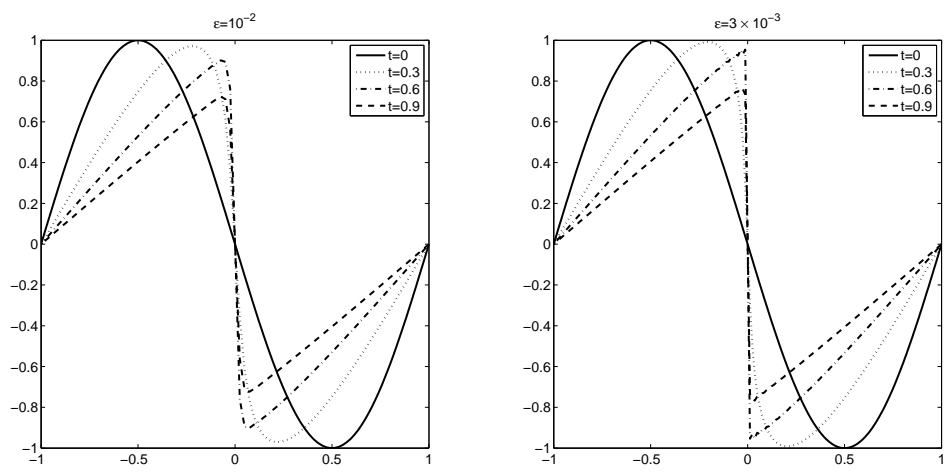

Figure 2: Numerical solutions for Example 4.1, at different times with viscosity $\epsilon=1 \mathrm{e}-2, M=127, \tau=0.1$ and $\epsilon=3 \mathrm{e}-3 \quad M=255, \tau=0.01$.

Table 8: Comparisons of Example 4.2 at different times for $\epsilon=0.01$.

\begin{tabular}{c|cc|cc|cc|cc}
\hline & \multicolumn{2}{|c|}{ Euler+BS [32] } & \multicolumn{2}{c|}{ RK4+BS } & \multicolumn{2}{c|}{ ECEM+BS } & \multicolumn{2}{c}{ ECEM+PS } \\
\hline & \multicolumn{9}{|c|}{$M=79$} & \multicolumn{2}{c}{$M=19$} \\
\hline$t$ & $E_{1}\left(t, \frac{1}{10^{4}}\right)$ & $\mathrm{cpu}$ & $E_{1}\left(t, \frac{5}{10^{3}}\right)$ & $\mathrm{cpu}$ & $E_{1}\left(t, \frac{4}{10^{2}}\right)$ & $\mathrm{cpu}$ & $E_{1}\left(t, \frac{1}{10}\right)$ & $\mathrm{cpu}$ \\
\hline 0.4 & $5.75 \mathrm{e}-3$ & 0.66 & $5.58 \mathrm{e}-3$ & 0.56 & $5.49 \mathrm{e}-3$ & 0.64 & $4.60 \mathrm{e}-3$ & 0.17 \\
0.6 & $1.21 \mathrm{e}-2$ & 0.73 & $1.20 \mathrm{e}-2$ & 0.57 & $1.21 \mathrm{e}-2$ & 0.68 & $8.48 \mathrm{e}-3$ & 0.18 \\
0.8 & $8.06 \mathrm{e}-3$ & 0.80 & $8.05 \mathrm{e}-3$ & 0.57 & $8.05 \mathrm{e}-3$ & 0.74 & $5.06 \mathrm{e}-3$ & 0.18 \\
1.0 & $4.72 \mathrm{e}-3$ & 0.88 & $4.74 \mathrm{e}-3$ & 0.57 & $4.74 \mathrm{e}-3$ & 0.78 & $2.66 \mathrm{e}-3$ & 0.18 \\
3.0 & $1.59 \mathrm{e}-4$ & 1.53 & $1.80 \mathrm{e}-4$ & 0.64 & $1.81 \mathrm{e}-4$ & 1.16 & $1.47 \mathrm{e}-4$ & 0.22 \\
\hline
\end{tabular}

Table 9: Comparisons of Example 4.2 at time 0.6 for $\epsilon=0.01$.

\begin{tabular}{c|ccc|ccc}
\hline & \multicolumn{3}{|c|}{ RK4+PS [21] } & \multicolumn{3}{c}{ ECEM+PS } \\
\hline$M$ & $\tau$ & $E_{1}(0.6, \tau)$ & $\mathrm{cpu}$ & $\tau$ & $E_{1}(0.6, \tau)$ & $\mathrm{cpu}$ \\
\hline 19 & 0.0025 & $7.70 \mathrm{e}-3$ & 0.17 & 0.05 & $7.70 \mathrm{e}-3$ & 0.18 \\
24 & 0.0025 & $7.64 \mathrm{e}-4$ & 0.22 & 0.02 & $7.65 \mathrm{e}-4$ & 0.26 \\
39 & 0.0005 & $1.11 \mathrm{e}-5$ & 0.39 & 0.02 & $1.04 \mathrm{e}-5$ & 0.51 \\
79 & 0.000025 & $3.56 \mathrm{e}-6$ & 3.92 & 0.01 & $3.56 \mathrm{e}-6$ & 1.92 \\
\hline
\end{tabular}



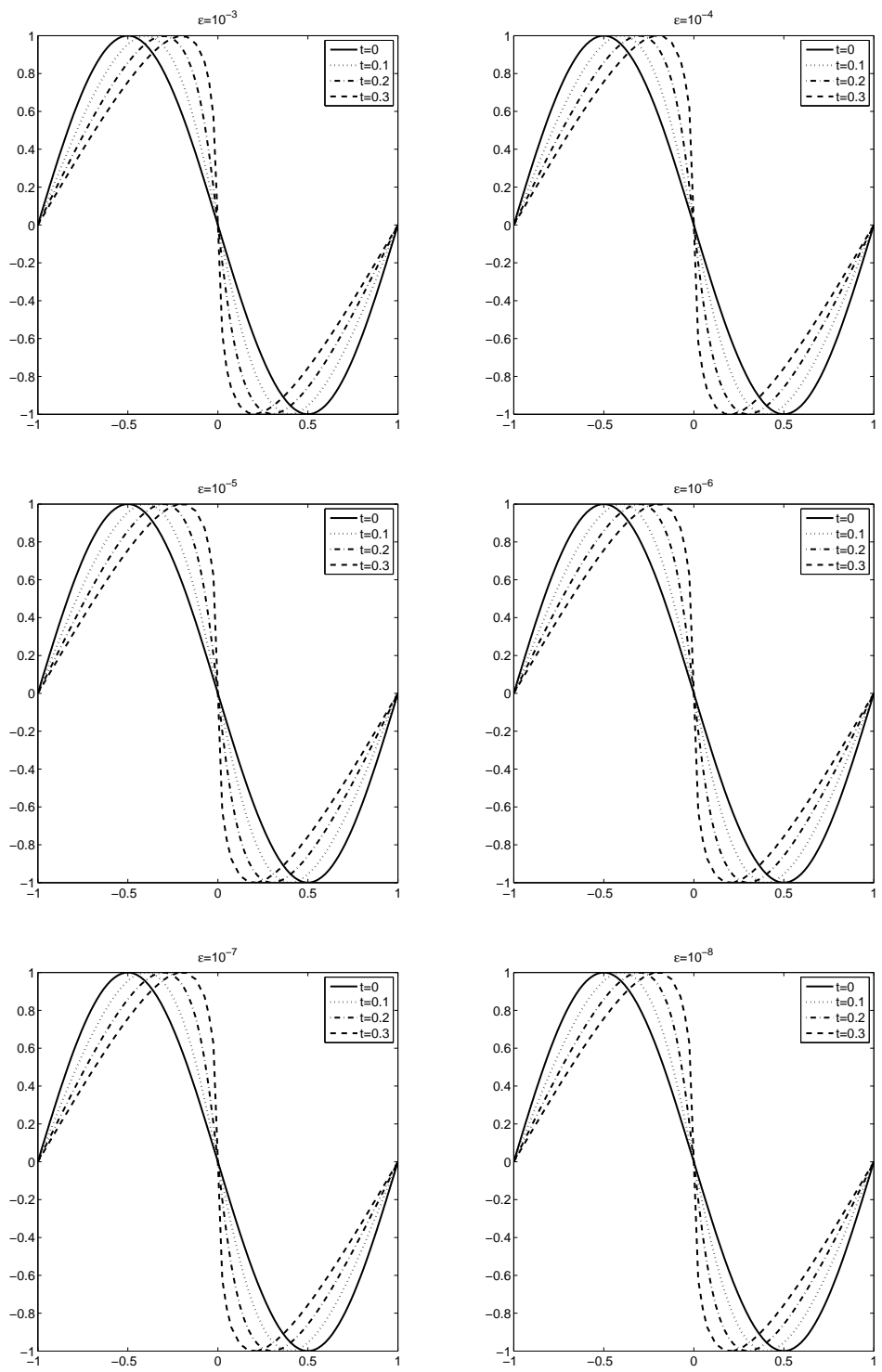

Figure 3: Numerical solutions for Example 4.1, at different times with small viscosity numbers when $M=127, \tau=0.1$. 
points $M$, we measure them under similar errors at the same time and space points. ECEM needs $\tau=0.01$ and $M=11$ but [16] and [28] require $M=79, \tau=0.001$ and $M=79, \tau=0.00001$ respectively. Even if ECEM uses the pseudo-spectral method for the space direction, it is remarkable phenomenon that ECEM works very well for the large time length $\tau=0.01$ compared with $\tau=0.001,0.00001$ for methods in [16] and [28]. One may see similar phenomena in table 7 for $\epsilon=0.01$ in which ECEM with pseudo-spectral method (ECEM+PS) is compared with the numerical results by finite difference in [23]. As one may guess, ECEM+PS yields more accurate solutions.

Table 8 explains both advantage and disadvantage about ECEM with the modified B-spline approximation and the pseudo-spectral approximation for the spatial discretization. For the B-spline approximation (BS), the required cpu time to get the similar maximum error for three time discretizations, Euler method ([32]), RK4 and ECEM are measured at different times. Even if ECEM and RK4 are fourth order convergence in time, ECEM allows much larger time step $\tau=0.04$ than $\tau=0.005$ while RK4+BS spends less cpu time than ECEM+BS does for the same accuracy under $\epsilon=0.001$ and $M=79$. The numerical results show that the combination ECEM+PS is much better than other combinations in the sense of the computational time (cpu-time) required in obtaining a similar maximum error. One may see similar phenomena in table 9 for $\epsilon=0.01$ at time $t=0.6$ in which ECEM with pseudo-spectral method (ECEM+PS) is compared with the numerical results by RK4 with pseudo-spectral method (RK4+PS) in [21]. As one may guess, ECEM+PS requires less computational time to get an accurate solutions.

Example 4.3. Consider the Burgers' equation $(2.1)$ on the interval $(0,1)$ with the non-periodic solution [3]

$$
u(t, x)=\frac{\sum_{k=1}^{3} 2 \sigma_{k} \exp \left(\frac{-\sigma_{k}\left(x-\eta_{k}-\sigma_{k} t\right)}{\epsilon}\right)}{\sum_{k=1}^{3} \exp \left(\frac{-\sigma_{k}\left(x-\eta_{k}-\sigma_{k} t\right)}{\epsilon}\right)},
$$

where $\sigma_{1}=0.05, \sigma_{2}=0.25, \sigma_{3}=0.5$ and $\eta_{1}=\eta_{2}=\eta_{3}=0.5$, for which the initial and boundary conditions are prescribed on all boundaries and the initial according to $(4.3)$.

For this example, two errors $E_{1}(t, \tau)$ and $E_{2}(t, \tau)$ are reported in Table 10. These errors from $t=0.2$ to $t=1.0$ seem to be bounded by $O\left(10^{-11}\right)$ or increased slightly when the fixed time length $\tau=0.1$ and $M=15$ are used for a slightly large viscosity $\epsilon=0.1$. One may need to investigate such error behaviors for a long time. According to Table 11, when the total number of points $M$ is increased from 15 to 255 for the fixed time length $\tau=0.1$, two errors are decreasing from $O\left(10^{-3}\right)$ to $O\left(10^{-12}\right)$ or $O\left(10^{-11}\right)$ at the time $t=1$ for the case $\epsilon=0.01$. These situation can be explained with the property of pseudo-spectral discretizations for the space direction. 
Table 10: Two error norms by ECEM at different times for Example 4.3 when $\epsilon=0.1, \tau=0.1, M=15$.

\begin{tabular}{cccccc}
\hline$t$ & 0.2 & 0.4 & 0.6 & 0.8 & 1.0 \\
\hline$E_{1}(t, \tau)$ & $1.83 \mathrm{e}-011$ & $5.84 \mathrm{e}-011$ & $3.67 \mathrm{e}-011$ & $2.99 \mathrm{e}-011$ & $3.21 \mathrm{e}-011$ \\
$E_{2}(t, \tau)$ & $4.43 \mathrm{e}-011$ & $1.24 \mathrm{e}-010$ & $7.20 \mathrm{e}-011$ & $5.75 \mathrm{e}-011$ & $6.05 \mathrm{e}-011$ \\
\hline
\end{tabular}

Table 11: Two error norms by ECEM at time $t=1$ for Example 4.3 when $\epsilon=0.01, \tau=0.1$.

\begin{tabular}{cccccc}
\hline$M$ & 15 & 33 & 63 & 127 & 255 \\
\hline$E_{1}(t, \tau)$ & $6.49 \mathrm{e}-003$ & $3.32 \mathrm{e}-005$ & $2.74 \mathrm{e}-009$ & $6.87 \mathrm{e}-012$ & $6.55 \mathrm{e}-012$ \\
$E_{2}(t, \tau)$ & $9.03 \mathrm{e}-003$ & $8.46 \mathrm{e}-005$ & $8.67 \mathrm{e}-009$ & $1.87 \mathrm{e}-011$ & $2.51 \mathrm{e}-011$ \\
\hline
\end{tabular}

Example 4.4[9, p. 119]. Consider the Burgers' equation (2.1) with $\epsilon=0.01$ on the interval $(-1,1)$ with the non-periodic solution

$$
u(t, x)=1+u_{b}(t+1, x-t)
$$

obtained by the Hopf-Cole transformation [13] $u_{b}(t, x)=-2 \epsilon \phi_{x} / \phi$, where $\phi(t, x)$ is given by

$$
\phi(t, x)=1+\sqrt{\frac{16}{t}} \exp \left(-\frac{x^{2}}{4 \epsilon t}\right) .
$$

Two errors $E_{1}(t, \tau)$ and $E_{2}(t, \tau)$ at time $t=1$ by ECEM and CCM [29] are displayed in Fig. 4. The left of Fig. 4 is obtained with $M=127$ and the several time lengths from $2^{-4}$ to $2^{-11}$. On the other hand, the right of Fig. 4 is obtained for the time length $\tau=0.001$ and several numbers $M$ from $2^{4}-1$ to $2^{8}-1$. Two figures in Fig. 4 show that both methods ECEM and CCM have similar convergence properties.

In Fig. 5, we compare the computational efficiencies of ECEM and CCM in terms of cpu time and feval for $M=127$ and $\tau$ from $2^{-4}$ to $2^{-11}$. The figures show that ECEM is superior to CCM because CCM requires Newton iteration process at each time step to solve the nonlinear system of ODEs (2.5), while ECEM needs only solving one linear system for whole process.

\section{Conclusion}

A new time stepping method called the error corrected Euler method (ECEM) for solving Burgers' equations is developed using the pseudo-spectral method for the 

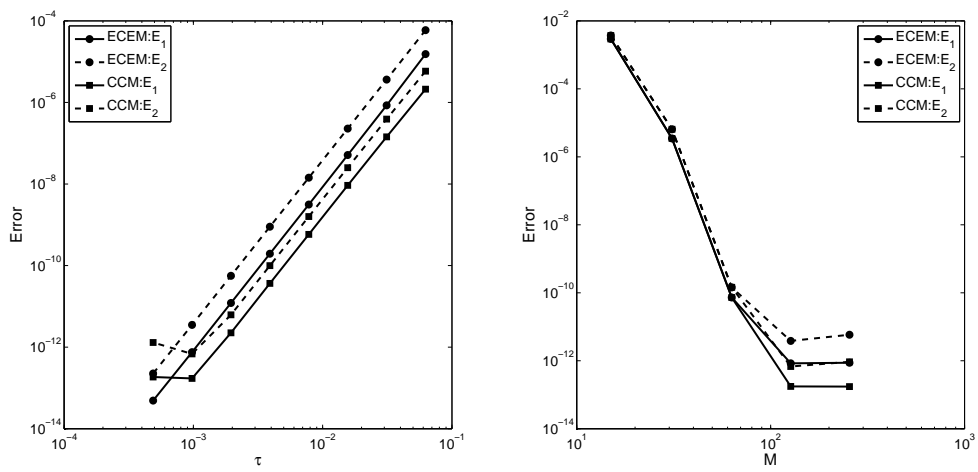

Figure 4: Computed two errors, $E_{1}(t, \tau)$ and $E_{2}(t, \tau)$, at time $t=1$ for Example 4.4.
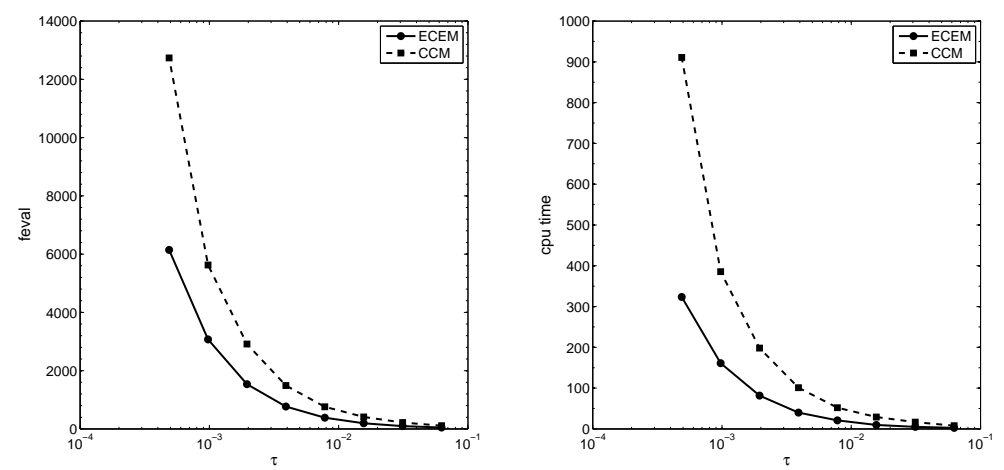

Figure 5: Comparison of feval(left) and cpu time(right) between the ECEM4 and CCM4 [29] with variable $\tau$ and fixed $M=127$ for Example 4.5. 
spatial discretization and ECEM for the time space. Throughout several numerical simulations, it is shown that the present method not only gives a good convergence behavior but also avoids unnecessary Newton iterations for the nonlinear system. Also, it is shown that the proposed method has a good numerical performance compared with other existing methods for several test problems. In particular, the present method radically reduce about one-half of the computational costs compared with existing implicit type methods. An interest remaining challenges in the proposed method is to develop a way to control the time step length to reduce the computational cost. In a forthcoming work, we deal with these topics with some applications to several other time dependent partial differential equations including two-dimensional Burgers' equations.

\section{References}

[1] A. H. A. Ali, G. A. Gardner, L. R. T. Gardner, A collocation solution for Burgers' equation using cubic B-spline finite elements, Comput. Methods Appl. Mech. Engrg., 100(1992), 325-337.

[2] A. R. Bahadir, A fully implicit finite-difference scheme for two-dimensional Burgers' equations, Appl. Math. Comput., 137(2003), 131-137.

[3] M. Berzins, Global error estimation in the methods of lines for parabolic equations, SIAM J. Sci. Statist. Comput., 19(4)(1988), 687-701.

[4] K. Black, A spectral element technique with a local spectral basis, SIAM J. Sci. Comput., 18(1997), 355-370.

[5] D. T. Blackstock, Convergence of the Keck-Boyer perturbation solution for plane waves of finite amplitude in vicous fluid, J. Acoust. Soc. Am., 39(1966), 411-413.

[6] N. Bressan, A. Quarteroni, An implicit/explicit spectral method for Burgers' equation, Calcolo, 23(1987), 265-284.

[7] J. M. Burgers, A mathematical model illustrating the theory of turbulence, Adv. Appl. Mech., 1(1948), 171-199.

[8] J. Caldwell, P. Wanless and A. E. Cook, A finite element approach to Burgers' equation, Appl. Math. Modelling, 5(1981), 189-193.

[9] C. Canuto, M. Y. Hussaini, A. Quarteroni and T. A. Zang, Spectral Methods: Fundamentals in Single Domains, Springer-Verlag, Berlin Heidelberg, 2006.

[10] H. Chen, Z. Jiang, A characteristics mixed finite element method for Burgers' equation, J. Appl. Math. Comput., 15(2004), 29-51.

[11] I. Christie, A. R. Mitchell, Upwinding of high order Galerkin methods in conductionconvection problems, Int. J. Numer. Methods Eng., 12(1978), 1764-1771.

[12] M. Ciment, S. H. Leventhal and B. C. Weinberg, The operator compact implicit method for parabolic equations, J. Comput. Phys., 28(1978), 135-166.

[13] J. D. Cole, On a quasi-linear parabolic equation occuring in aerodynamics, Quart. Appl. Math., IX, (1951), 225-236. 
[14] G. Dahlquist, A special stability problem for linear multistep methods, BIT, 3(1963), $27-43$.

[15] M. O. Deville, P. F. Fischer and E. H. Mund, High-order methods for incompressible fluid flow, Cambridge University Press, New York, 2002.

[16] I. A. Hassanien, A. A. Salama and H. A. Hosham Fourth-order finite difference method for solving Burgers' equation, Applied Math. and Comput., 170(2005), 781-800.

[17] B. M. Herbst, S. W. Schoombie, D. F. Griffiths and A. R. Mitchell, Generalized Petrov-Galerkin methods for the numerical solution of Burgers' equation, Int. J. Numer. Methods Eng., 20(1984), 1273-1289.

[18] R. S. Hirsh, Higher order accurate difference solutions of fluid mechanics problems by a compact differencing technique, J. Comput. Phys., 19(1975), 90-105.

[19] A. N. Hrymak, G. J. Mcrae and A. W. Westerberg, An implementation of a moving finite element method, J. Comput. Phys., 63(1986), 168-190.

[20] P. Z. Hunag, A. Abduwali, The modified local Crank-Nicolson method for one- and two-dimensional Burgers' equations, Compu. Math. Appl., 59(2010), 2452-2463.

[21] A. H. Khater, R. S. Temsah and M. M. Hassan, A Chebyshev spectral collocation method for solving Burgers'-type equations, J. Comput. Appl. Math., 222(2008), 333350 .

[22] P. Kim, X. Piao and S. Kim, An error corrected Euler method for solving stiff problems based on Chebyshev collocation, SIAM J. Numer. Anal., 49(2011), 2211-2230.

[23] S. Kutluay, A. R. Bahadir and A. Özdeş, Numerical solution of one-dimensional Burgers equation: explicit and exact-explicit finite difference methods, J. Comput. and Applied Math., 103(1999), 251-261.

[24] S. Kutluay, A. Esen and I. Dag, Numerical solutions of the Burgers' equation by the least-squares quadratic B-spline finite element method, J. Comput. Appl. Math., 167(2004), 21-33.

[25] M. J. Lighthill, 'Viscosity Effects in Sound Waves of Finite Amplitude', In: Surveys in Mechanics, ed. by G.K. Bauchelor, R. Davies, Combridge Univ. Press, 1956.

[26] R. C. Mittal, R. Jiwari, Differential Quadrature Method for Two-Dimensional Burgers' equations, Int. J. Comput. Methods Eng. Sci. Mech., 10(2009), 450-459.

[27] A. R. Mitchell, D. F. Griffiths, The finite difference method in partial differential equations, John Wiley \& Sons, New York, 1980.

[28] T. Öziş, E. N. Aksan and A. Özdes, A finite element approach for solution of Burgers' equation, Applied Math. and Comput., 139(2003), 417-428.

[29] H. Ramos, J. Vigo-Aguiar, A fourth-order Runge-Kutta method based on BDF-type Chebyshev approximations, J. Comput. Appl. Math., 204(2007), 124-136.

[30] L. N. Trefethen, Spectral Methods in MATLAB, Software Environ. Tools 10, SIAM, Philadelphia, 2000.

[31] Y. Wu, X. H. Wu, Linearized and rational approximation method for solving nonlinear Burgers' equation, Int. J. Numer. Methods Fluids, 45(2004), 509-525.

[32] M. Xu, R. H. Wang, J. H. Zhang and Q. Fang, A novel numerical scheme for solving Burgers' equation, Applied Math. and Comput., 217(2011), 4473-4482. 
[33] L. Zhang, J. Ouyang, X. Wang and X. Zhang, Varational multiscale element-free Galerkin method for 2D Burgers' equation, J. Comput. Phys., 229(2010), 7147-7161. 